\title{
Synergy between medicinal chemistry and biological research
}

Interview by Hannah Coaker (Commissioning Editor, Future Science Group).

Salvador Moncada studied medicine at the University of El Salvador (El Salvador) before coming to the UK in 1971 to work on a PhD with Professor John Vane at the Institute of Basic Medical Sciences, Royal College of Surgeons (UK). After a short period of research at the University of Honduras (Honduras), he joined the Wellcome Research Laboratories (UK) where he became Head of the Department of Prostaglandin Research and later, Director of Research. He returned to academic life in 1996 as founder and director of the Wolfson Institute for Biomedical Research at University College London (UK). Moncada played a role in the discovery of the mechanism of action of aspirin-like drugs and later led the teams which discover prostacyclin and identified nitric oxide as a biological mediator. In his role as a Director of Research of the Wellcome Laboratories, he oversaw the discovery and development of medicines for epilepsy, migraine, malaria and cancer. Currently, he is working on the regulation of cell proliferation as Director of the Institute of Cancer Sciences at the University of Manchester (UK). Moncada has won numerous awards from the international scientific community and in 2010, he received a knighthood from Her Majesty Queen Elizabeth II for his services to science.

Q How did you become involved in the field of medicinal chemistry?

When I was appointed Director of Research at the Wellcome Research Laboratories, there was a large department of medicinal chemistry with excellent scientists reporting to me. I became fascinated by the strength and scope that chemistry can add to biological research.

Q Can you update us on some of your research group's current work? Is there an area of your research that you are particularly excited about at the moment?

In the last few years, I have been interested in the metabolism of proliferating cells. Abnormal cell proliferation is a hallmark of cancer. The questions that interest me are:

"Is the metabolism of highly proliferative cancer cells similar or different to that of normal cells which are proliferating?"
"If it is different, how different is it?" "Are any such differences sufficiently significant to allow a medicinal chemistry approach toward selective inhibition of cancer cell proliferation?"

I am most excited about the possibility of finding a small molecule that will target selectively the metabolism of cancer cells.

Q How has your medicinal chemistry background prepared you for your current, more clinical role?

I have learned that medicinal chemistry is an invaluable tool for investigating biological processes by using small molecules as probes. Some probes go into further development as projects for drug discovery and some of those become drugs. During my years as Director of Research at Wellcome, we developed a number of new medicines, including lamotrigine for epilepsy, zomig for migraine

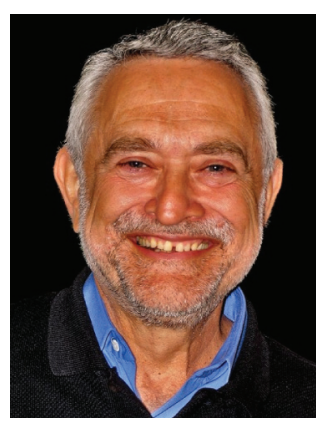

Salvador Moncada Director of Cancer Sciences, University of Manchester, Wolfson Molecular Imaging Centre, 27 Palatine Road, Manchester M20 3LJ, UK salvador.moncada@manchester.ac.uk 
and atovaquone for malaria; we also initiated the project that led to the discovery of lapatinib for cancer. Medicinal chemistry has also been very important for the pursuit of my research.

Q An important milestone in your career was the discovery of prostacyclin. Can you describe the findings that led to this discovery?

We found prostacyclin shortly after we discovered thromboxane A2synthae in the platelets. I was interested in investigating the generation of this powerful vasoconstrictor and inducer of platelet aggregation in the vasculature. To our great surprise, we found that the vessel wall does not make thromboxane $\mathrm{A} 2$ but instead makes the biological opposite - a powerful vasodilator and inhibitor of platelet aggregation. This became known as prostacyclin. From that discovery, we developed the concept that there is in the vascular system a balance between prostacyclin and thromboxane A2, the disruption of which leads either to beneficial effects or to pathophysiology.

Q You were a key player in demonstrating that endothelium-derived relaxing factor (EDRF) was in fact nitric oxide (NO) and identifying this as a signaling molecule in the cardiovascular system - can you explain what led you and your team to make this breakthrough?

Following Furchgott's discovery of EDRF, we found that this compound was probably a free radical, sensitive to the destructive action of superoxide anions. We developed a new methodology to study the nature of this unidentified substance and produced the earliest evidence in favor of the hypothesis that EDRF was NO. We further showed that NO is released by the vascular endothelium in quantities sufficient to explain the biological activity of EDRF.

Q In your opinion, what impact have these discoveries made on medical science and how have they shaped your career?

The discovery of both prostacyclin and nitric oxide has been very important for the understanding of the vascular system. NO plays a fundamental role in the regulation of normal blood flow and blood pressure but, if released in abnormal quantities, is involved in pathophysiological conditions such as septic shock. Furthermore, our knowledge of the balance between prostacyclin and thromboxane A2 led to the understanding of how small doses of aspirin protect against cardiovascular disease. Currently, there are probably more than 200 million people taking a small dose of aspirin every day for this purpose. In contrast, the cardiovascular side effects of the newer COX-2 inhibitors are clearly attributable to inhibition of prostacyclin. My career in science has been heavily influenced by these discoveries, but they have only increased my interest in finding more new things.

\section{Q During your time in academic research} you have worked in close collaboration with pharmaceutical industry. In your experience, do you think that partnerships between academia and industry benefit the drug discovery process? Yes, it does! I have found the collaboration between academia and industry to be extremely useful and fruitful. To me, one of the most exciting steps in the drug development process is the stage at which a research idea is converted into a drug discovery program, which is nowadays called 'from the bench-to-the-clinic.' That is precisely the interface between academia and industry and is the point at which scientific and practical considerations are used in combination for decision making. I feel that, over the years, both industry and academia have recognized and developed successful interactions that are bound to improve with time. In addition, industry has understood the need to support fundamental research carried out in academia. This is the reason why more industrial funding is going into basic research in academic centers. This is a welcome development and will result in stronger and more strategic partnerships.

\section{Q How do you anticipate the field of cancer} research will progress in the next $5-10$ years? That is impossible to predict. Science often surprises us and we never know when a significant breakthrough will occur that will radically change our views. That said, I think that we will continue on the path of the development of small molecules and monoclonal antibodies that are targeted toward specific pathways. By using biomarkers among other things, we will understand better how to treat each patient individually. We will learn to combine different molecules more effectively and also to combine them with other forms of treatment such as radiation and/or immunotherapy.

We will also understand better the nature of the side effects these combinations produce and the best way to avoid or ameliorate them. All this will lead to a considerable lengthening of the time these patients can continue to live a normal life. I feel, however, that the most significant developments in the cancer field will be in prevention and early diagnosis. This is not only possible but most desirable, since early diagnosis is the key to successful management of cancer.

Q What words of advice do you have for a young scientist envisioning a career in cancer research? 
My advice to any young scientist is to do science only if you are passionate about it. If you are, the intense dedication that is required for success will be the most enjoyable experience.

\section{Disclaimer}

The opinions expressed in this interview are those of the interviewee and do not necessarily reflect the views of Future Science Ltd.

\section{Financial \& competing interests disclosure}

The author has no relevant affiliations or financial involvement with any organization or entity with a financial interest in or financial conflict with the subject matter or materials discussed in the manuscript. This includes employment, consultancies, honoraria, stock ownership or options, expert testimony, grants or patents received or pending or royalties.

No writing assistance was utilized in the production of this manuscript. 